\title{
Erratum
}

\section{Axilla surgery severely affects quality of life: results of a 5-year prospective study in breast cancer patients}

Jutta Engel ${ }^{1}$, Jacqueline Kerr ${ }^{1}$, Anne Schlesinger-Raab ${ }^{1}$, Hansjörg Sauer ${ }^{2}$, and Dieter Hölzel ${ }^{1}$ ${ }^{1}$ Munich Field Study, Munich Cancer Registry, ${ }^{2}$ Med. Klinik III, Klinikum Großhadern, Ludwig-MaximiliansUniversity, Munich, Germany

Breast Cancer Research and Treatment 79: 47-57, 2003

The values for 'Negative' and 'Positive' under 'Lymph nodes' in Table 1 (p. 49) of the original were presented the wrong way around. The right values are given below.

Negative $583(60.0)$

Positive $323(33.2)$ 